\title{
iOS System Based on Sina Microblogging Mobile APP Design \\ Haiyang $\operatorname{Lv}^{1, a^{*}}$ and Jingnan Liu ${ }^{1, b}$ \\ ${ }^{1}$ Yantai Nanshan University, Yantai 265713, China \\ alvhaiyang2012@sina.cn, bliujn0228@163.com
}

Keywords: iOS; IPhone; Mobile terminal; Sina microblogging

\begin{abstract}
The purpose of this project is to develop an operating system based on iOS Sina Weibo iOS client client software.This paper covers the development of Sina microblogging client some knowledge. The design with the Apple system to iOS as a system development and runtime environment, complete system design, development and testing. Xcode development environment, the use of Sina open platform API interface, data exchange can be provided either by way of using a web access, but also to achieve a variety of ways, and the site became a development platform launched microblogging client Development and implementation. The software microblogging Home Get Data, comment microblogging, forward microblogging, microblogging and other functions published, with the general micro-Bo has the function, practicality is also good.
\end{abstract}

\section{Development Background and Significance}

SNS(Social Networking Services) Social services using the characteristics of the network extension of real life social circle, which is very convenient and fast to build their own circle of friends. Weibo is a member of the SNS. The earliest and most famous Twitter microblogging is the United States. The vigor of Twitter is open API, Twitter's success and open apis are inseparable. This paper is to use sina weibo open API. In the age of the Internet, mobile phones become people to quickly get, distribution and transfer information important channel. To this end, I chose based on a development of the iOS app, with specific implementing a sina microblogging account, for the purpose of related work.

\section{Development Tools and Runtime Environment}

Development Tools. Xcode6.2 + iOS8.0 version is used for developing the software [1].Objective is the development of language - $\mathrm{C}$ to develop the software [2-3].

Runtime Environment. The version of the software for runningde mobile phone is iphone 4 and above version.

\section{Introduction of $\mathrm{iOS}$}

System Architecture of iOS. iOS is based on the UNIX, communicate directly with the underlying hardware. System underlying, application framework, software is the $\mathrm{C} / \mathrm{C}++$ or Objective - C, so has high efficiency, in terms of the stability of the system, much better than any other operating system.

iOS system structure is divided into the following four levels: the Core operating system (the Core OS layer), the Core Services layer (the Core Services layer), Media layer (the Media layer), Cocoa Touch framework layer (the Cocoa Touch layer). As shown in Fig. 1. 


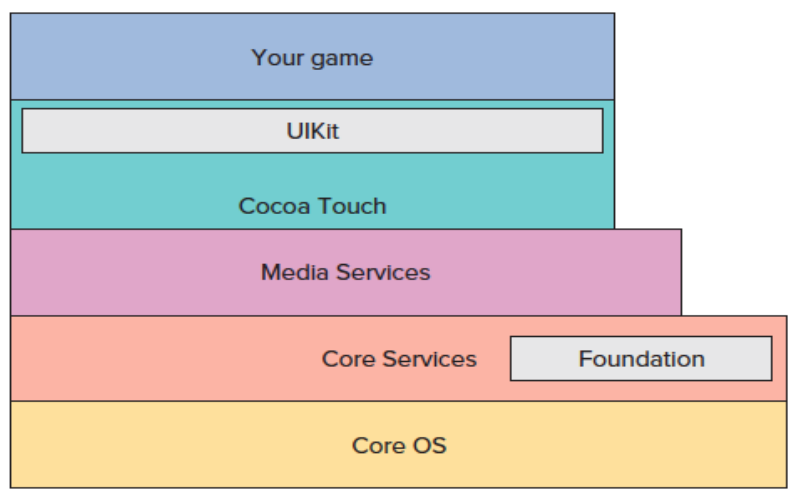

Figure 1. iOS System structure of hierarchy

Design Patterns of iOS. MVC (Modol View Controller) is a kind of the earliest and most successful reusable design patterns, the '70 s successful use in smaltalk program for the first time. Based on the MVC design pattern, the Cocoa overall architecture can be divided into three subsystems: Model, View and Controller.

Modol, View and Controller to understand the role of the role of each subsystem in the MVC design pattern, we need to understand the function and behavior of an application. The following is the role of MVC:

Model: It is used for data layer to store data. For the application to provide independent, independent of the View and Controller subsystem data model.

View: It is the view layer interface will be presented. Like in the form of a certain user to present data and allow users to manipulate, the data can be displayed in the list of, can be in the form of pictures, sometimes just show some data based on the requirements.

Controller: It is the controller is responsible for the connection between the data layer and view layer. Between the Model and the View of joining a Controller's main purpose is to "decoupling", which eliminate the coupling between the Model and the View. Users interact with the View, the View will notify the Controller in the View of the data has changed, and the Controller is the data change in response to the Model.

The decoupling Controller function is shown in Fig. 2 [12].
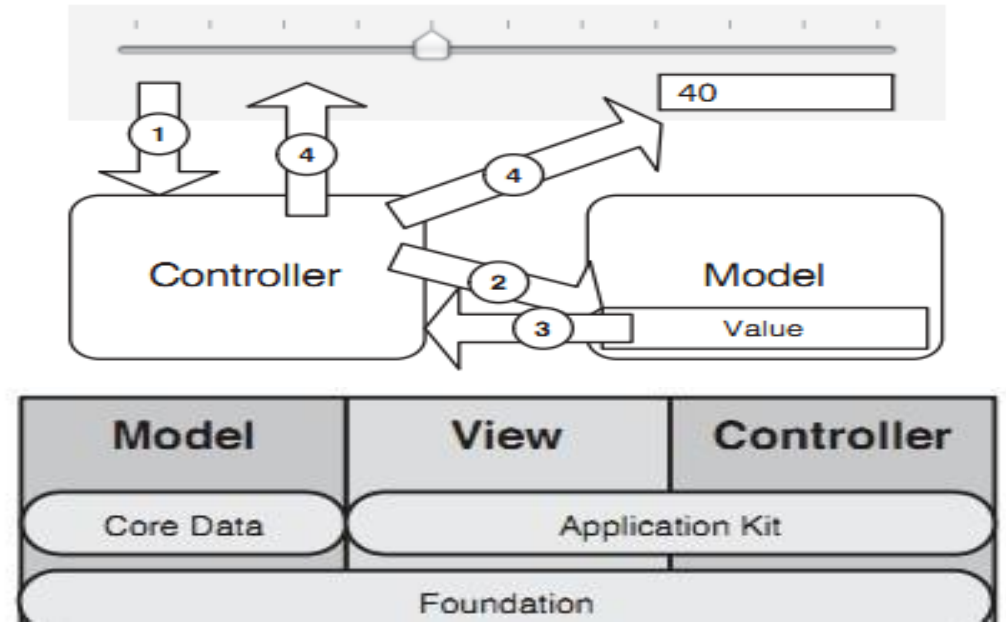

Figure 2. The decoupling of Controller

\section{System Design and Implementation}

Land Registration and Authorization Module. The user registration page: When a user first use this version and have not yet received the sina weibo account, skip to sina weibo user registration page. Builds with UI interface, set up two text box input required for mobile number and password, data request by post/get speak and fill in the content of the uploaded to interface to register. 
Third-party applications must first be registered on the sina weibo platform for the developers to be developers and obtain the corresponding key app and app secret can guide the user registration[6].The open platform of sina weibo website: http://open.weibo.com/。

Obtaining authorization token value: NSString *urlStr $=$ [kAuthorizeURL stringByAppendingFormat:@"?display=mobile\&client_id=\%@\&redirect_uri=\%@",kAppKey, kRedirectURI]; e

NSURL *url = [NSURL URLWithString:urlStr];

NSURLRequest $*$ request $=$ [NSURLRequest requestWithURL:url];

Login authorization page: For the first time to use this version or didn't remember the password and registered users of success, into the program enter the authorization of landing interface, enter the user name and password for authorization certification. Set up from the page text box can button controls, such as data request via post/get speak and fill in the content of the uploaded to the landing interface.

The First Interface Display Module. This page is the main interface software, in order to the user experience better not login registration screen also can enter the page, when you need the user login prompt the user login, only when the page is the main use of controls tableView, custom cell, and then to load the data in the tableView. On the interface of the refresh and drop-down load code for the: MJRefreshHeaderView *header = [MJRefreshHeaderView header $]$;

header.scrollView = self.tableView; header.delegate $=$ self;

[header beginRefreshing];

MJRefreshFooterView $*$ footer $=[$ MJRefreshFooterView footer $]$;

footer.scrollView = self.tableView;

footer.delegate $=$ self;

Check the Weibo Content Module.When entering the home page, under the list of weibo selected click a cell, you like into the weibo content module for details.

The key code for weibo details page:

if (indexPath.section $==0$ ) \{

static NSString *CellIdentifier = @ "DetailCell";

StatusDetailCell *cell = [tableView dequeueReusableCellWithIdentifier:CellIdentifier];

if $($ cell $==$ nil $)\{\quad$ cell $=[$ [StatusDetailCell alloc $]$

initWithStyle:UITableViewCellStyleDefault reuseIdentifier:CellIdentifier];

\}

cell.cellFrame =_detailFrame;return cell;

\}

static NSString *CellIdentifier = @ "Cell";

UITableViewCell *cell = [tableViewdequeueReusableCellWithIdentifier:CellIdentifier $]$;

if $($ cell $==$ nil $)\{$

cell $=[$ [UITableViewCell alloc $]$ initWithStyle:UITableViewCellStyleDefault

reuseIdentifier:CellIdentifier]; \}

Forward comment code

[self setBtn:_comment title:@" comment "count:status.commentsCount];

[self setBtn:_repost title:@" forward "count:status.repostsCount];

Published Weibo Module. When we enter weibo app, click on the plus sign at the bottom of the navigation ICONS, published in weibo page, here you can type you want to say, add your favorite picture or your favorite expression.

Check the User Information Module.Function description: the detailed information to see friends.The step conditions: user login successfully; Precondition, logged-in users; Postconditions, details see friends attention and cancel the operation. 


\section{The System Testing}

Unit Testing. The first step: in the test system is testing system of individual component. In the process of unit tests, first check code can run (in the data, the program of algorithm, and bad grammar have), finally to the component input a set of test cases, used to illustrate these artifacts are correct. In a unit test, the main function of a single module test, regardless of other coupling module. The first step

When user login authorization configuration, one user name or password is empty or fill in error, cause the user cannot login successfully, if and only if the user name and password are right to succeed and preset operating system is light up to alert.

Specific operation: user name, password, any item is empty or fill in the wrong.

Results: both the corresponding reason for the error message.

Conclusion: requires the user to input the correct user name and password, to be successful authorization

The second step: When published new weibo, enter empty content, or as a content on weibo, which weibo cannot successfully published, if there is a preset message application.

Specific operation: the content of the input is empty, or on the same content.

Results: the content of prompting the user can't be empty, or please don't input the same content.

Conclusion: ask for correct content.

The third step: Weibo that comment, the input and comment, if the content is empty, the comment is not successful, whether the program has a predefined content is empty.

The specific operation: enter empty comment.

Results: according to comment on content cannot be empty.

Conclusion: the microblog comment function is normal

The forth step: On a user or weibo attention and cancel on operating time, verify whether has been cancelled/has added to its attention.

Specific operation: click on the button and a cancel button.

Results: the state show concern or cancelled.

Conclusion: add the normal function of attention and cancel the attention.

The Conclusion of System Testing. Above is for the client's function test, a total of four, and test results are normal, the system is running good, general operation can realize user functions. But there are many places of imperfect and system, need to process after the relay continued to improve and reconstruct unceasingly, improving performance and stability.

\section{Conclusion}

Based on the above design iOS function of sina weibo are realized. Weibo is a big platform, it contains many functions, can accommodate more personalized design, due to the personal skill level and time limit, the design of this system mainly lies in the list of weibo, details of the function module to realize the basic function such as published, on the basis of the realization of the function of a lot remains to be further optimized, but the design of this system can accommodate more personalized design, the system structure more flexible, is advantageous to the development and the development of the follow-up. Only by constantly innovation, to create infinite value.

\section{References}

[1] Y.H.Zhang: iOS development series C/Objective-C language foundation. (Beijing people's press, Chinese 2011), p.101. (In Chinese)

[2] x.Liu: iOS development series iOS fifth edition in the Development guides. (Beijing people's publishing house, Chinese 2012), p.82. (In Chinese)

[3] Dave Mark, Jack Nutting and Jeff LaMarche: iPhone4 and iPad development of basic tutorial(People's posts press ,Chinese 2012), p.224. 
[4] Information on http://open.weibo.com/wiki/\%E5\%BE\%AE\%E5\%8D\%9AAPI [EB/OL].

[5] Information on, http://open.weibo.com/development/mobile[EB/OL].

[6] Y.WANG: iOS platform client application development standardization research (MS, BeiJing Post University, China 2013), p.28. (In Chinese).

[7] Carlo Chung: Objective-C the tao of programming :iOS design pattern parsing(People's posts press, Chinese 2012), p.108.

[8] Erik M.Buck and Donald A.Yacktman: Cocoa Design Pattern (Mechanical press, Chinese 2013), p.67.

[9] D.S.Guan: Basis from zero to App Store shelves (People's posts press, Chinese 2015), p.99.

[10]Natiuise: Proficient in iOS development (People's posts press, Chinese 2014), p.126.

[11]Q.Tang: iOS development advanced (Electronic industry press, Chinese 2014), p.145. (In Chinese) 